(C) 2005 The Japan Society of Applied Physics

\title{
High-Performance Narrow-Bandwidth Multicolor InAs/AIGaAs/GaAs Quantum Dot Infrared Photodetector
}

\author{
Shen-De Chen, Ying-Ying Chen and Si-Chen LeE* \\ Department of Electrical Engineering, Graduate Institute of Electronics Engineering, National Taiwan University, \\ Taipei, Taiwan, Republic of China
}

(Received November 16, 2004; accepted March 28, 2005; published August 5, 2005)

By introducing a $2 \mathrm{~nm} \mathrm{Al}_{0.3} \mathrm{Ga}_{0.7}$ As cap layer on InAs/GaAs quantum dots, a high-performance narrow-bandwidth multicolor quantum dot infrared photodetector (QDIP) with peak responses at 5.4, 9.5, and $15.8 \mu \mathrm{m}$ was fabricated successfully. The highest peak responsivities reached $0.79,0.47$, and $0.63 \mathrm{~A} / \mathrm{W}$ at a bias of $-1.2 \mathrm{~V}$. The two shorter-wavelength responses originating from two different-sized InAs quantum dots were due to the transitions of InAs quantum dots from the ground state to the GaAs conduction band. The separation of the two peaks was amplified by the insertion of the $2 \mathrm{~nm}$ AlGaAs cap layer. The longer-wavelength peak at $15.8 \mu \mathrm{m}$ was due to the transition from the ground state to the first excited state followed by the tunneling out of small quantum dots. The increase in photocarrier lifetime by the barrier is proposed to explain the very high responsivity. [DOI: 10.1143/JJAP.44.6307]

KEYWORDS: InAs, quantum dot infrared photodetector, responsivity, photoluminescence, solid source MBE

\section{Introduction}

Self-assembled InAs quantum dots on a GaAs substrate prepared by molecular beam epitaxy (MBE) have attracted much attention in recent years. ${ }^{1-5)}$ Due to its long capture and relaxation times, ${ }^{6}$ ) the QD structure is suitable for optoelectronic applications in infrared photodetectors, ${ }^{7-10)}$ lasers, ${ }^{11,12)}$ and optical memories. ${ }^{13)}$ Due to the threedimensional (3D) confinement of carriers, a quantum dot infrared photodetector (QDIP) exhibits improved device performances, such as a low dark current and a higher operating temperature. ${ }^{7,9,10)}$ However, unlike a quantum well infrared photodetector (QWIP) or a superlattice infrared photodetector (SLIP), the QDIP exhibits a broadband response $\mathrm{r}^{7-10,14)}$ from 3 to $12 \mu \mathrm{m}$ because of a wide distribution of dot size. Krishna et al. ${ }^{15}$ ) have reported a three-color InAs/InGaAs quantum-dots-in-a-well detector whose peaks locate at $3.8,8.5$, and $23.2 \mu \mathrm{m}$ with responsivities less than $25 \mathrm{~mA} / \mathrm{W}$ at $4.6 \mathrm{~K}$. The responsivities of 3.8 and $8.5-\mu \mathrm{m}$ peaks are only several $\mathrm{mA} / \mathrm{W}$, and their linewidth ratios, which are defined to be the full width half maximum over peak wavelength $(\Delta \lambda / \lambda)$, are as large as 42 and $28 \%$, respectively. The linewidth ratio of the $23.2 \mu \mathrm{m}$ peak is also large. The quantum-dots-in-a-well structure fabricated using a $\operatorname{In}_{0.15} \mathrm{Ga}_{0.85}$ As layer can decrease energy states, but there is no sufficiently high barrier that blocks dark current and improve the performance of QDIPs. In this paper, we propose a different QDIP structure fabricated by capping the InAs QDs with a thin AlGaAs layer. The $\mathrm{AlGaAs}$ layer can enhance carrier confinement. Compared with the structure proposed by Krishna et al. ${ }^{15)}$ this InAs/ AlGaAs/GaAs QDIP exhibits multiple response peaks with smaller linewidth ratios of 29, 13 and $8 \%$, and an extremely high responsivity of $0.79 \mathrm{~A} / \mathrm{W}$. The origins of these peaks are also explained.

\section{Experiments}

The InAs/AlGaAs/GaAs QDIP, as shown in Fig. 1, was grown using a VG solid-source molecular beam epitaxy (MBE) machine on a semi-insulating GaAs(100) substrate.

*E-mail address: sclee@cc.ee.ntu.edu.tw
The ten-stacked active region was grown at $510^{\circ} \mathrm{C}$. Every stack consisted of three-monolayer (3-ML) Si-doped InAs QDs covered with $2 \mathrm{~nm} \mathrm{Al}_{0.3} \mathrm{Ga}_{0.7} \mathrm{As}$ and $30 \mathrm{~nm} \mathrm{GaAs}$ spacers. From an atomic force microscope (AFM) image, which is not shown here, the dot density was about $4 \times 10^{10}$ $\mathrm{cm}^{-2}$. The dopant concentration in InAs QDs was $1 \times 10^{18}$ $\mathrm{cm}^{-3}$, which was estimated to be one $\mathrm{Si}$ atom per dot. After the growth of InAs QDs, all shutters were closed for $5 \mathrm{~s}$ to allow atoms to migrate on the surface. Finally, the $100 \times$ $100 \mu \mathrm{m}^{2}$ device was fabricated. The responsivity of the device was measured under an $45^{\circ}$-edge-coupling scheme with closed-cycle cryogenics and Perkin-Elmer S2000 Fourier transform infrared spectroscopy. Absolute spectral responsivity was calibrated with a standard blackbody radiation source operated at $1080 \mathrm{~K}$. For photoluminescence (PL) measurements, the samples were mounted in a closedcycle He cryostat, which provides the lowest temperature of $11 \mathrm{~K}$. PL was excited using an $\mathrm{Ar}^{+}$laser at a $514.5 \mathrm{~nm}$ wavelength and spot size of $300 \mu \mathrm{m}$. Finally, PL through a monochromator was detected using a InGaAs detector whose detection range was below $1.7 \mu \mathrm{m}$.

\section{Results and Discussion}

Figure 2 shows the spectral response of the 3-ML InAs/ $\mathrm{AlGaAs} / \mathrm{GaAs}$ QDIP under different biases at $20 \mathrm{~K}$; the inset displaying the spectra of a conventional 3-ML InAs/GaAs QDIP without the AlGaAs cap layer is shown for comparison. Three large peaks at $5.4,9.5$, and $15.8 \mu \mathrm{m}$ are clearly observed. One medium peak at $6.4 \mu \mathrm{m}$ and one small peak at $18 \mu \mathrm{m}$ are also observed. It appears that the three peaks at 5.4, 6.4 and $9.5 \mu \mathrm{m}$ form a group with a similar transition mechanism, whereas the two peaks at 15.8 and $18 \mu \mathrm{m}$ form another group. The linewidth ratios $(\Delta \lambda / \lambda)$ of the three large peaks are $0.29,0.13$, and 0.08 . Their responsivities under a $-1.2 \mathrm{~V}$ bias are very high, i.e., 0.79, 0.47, and $0.63 \mathrm{~A} / \mathrm{W}$. On the other hand, the inset represented an InAs/ GaAs QDIP with a single broadband response from 3 to $12 \mu \mathrm{m}$ under a $-1 \mathrm{~V}$ bias. Note that the peak responsivity of the InAs/GaAs QDIP reaches its maximum under the $-1 \mathrm{~V}$ bias. Then the spectrum becomes noisy and no peak is observed under the $-1.2 \mathrm{~V}$. This indicates that the additional $2 \mathrm{~nm} \mathrm{Al} \mathrm{Al}_{0.3} \mathrm{Ga}_{0.7} \mathrm{As}$ capping layer on InAs QDs resulted in the 


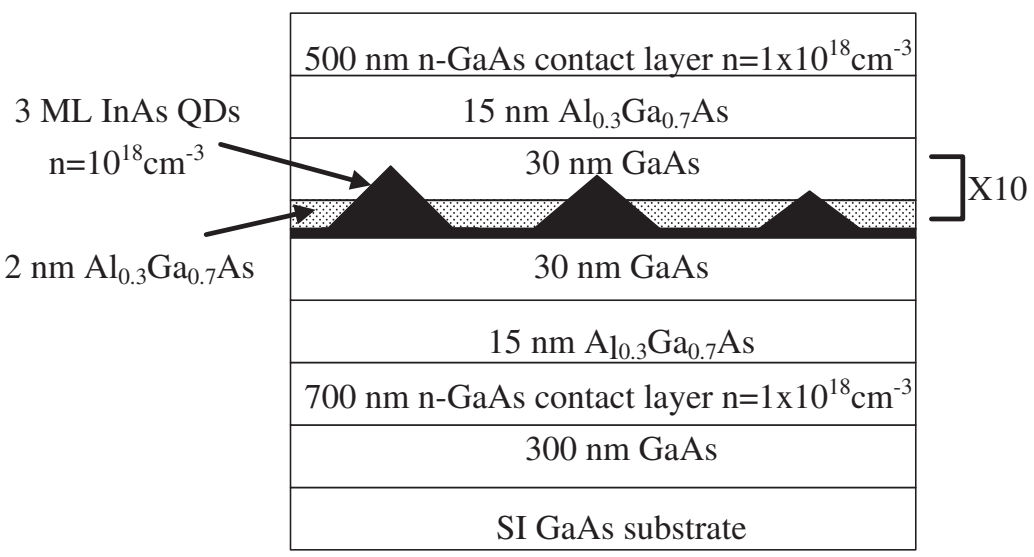

Fig. 1. Schematic device structure of InAs/AlGaAs/GaAs QDIP.

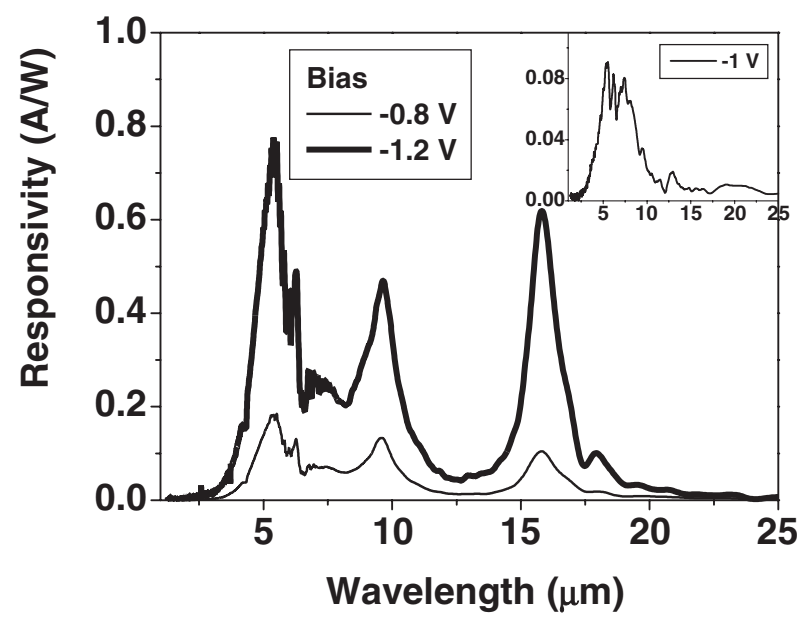

Fig. 2. Spectral response of InAs/AlGaAs/GaAs QDIP under biases of -0.8 and $-1.2 \mathrm{~V}$. The inset shows the spectral response of a conventional InAs/GaAs QDIP under $-1 \mathrm{~V}$ bias.

narrowing and separation of the broadband response into three distinct peaks and two extra long-wavelength peaks. To determine the role of the thin $\mathrm{Al}_{0.3} \mathrm{Ga}_{0.7} \mathrm{As}$ capping layer in the QDIP, power-dependent PL spectra are measured.

Figure 3(a) shows the PL spectrum fitted with three Gaussian peaks and the inset displays the power-dependent spectra at various powers from 8 to $300 \mathrm{~mW}$, i.e., the power density varies from 11 to $424 \mathrm{~W} / \mathrm{cm}^{2}$. The three peaks with different strengths located at 1.074 (peak 1), 1.113 (peak 2), and 1.179 (peak 3) eV. If peaks 1, 2 and 3 were due to the ground, first and second excited state transitions, respectively, the relative PL intensities of the higher energy peak would increase with laser power due to the state-filling effect. ${ }^{16,17)}$ Figure $3(\mathrm{~b})$ shows the PL intensity ratio, i.e., peak 2 /peak 1 , peak 3 /peak 1 , as a function of laser power. It is clear that the ratio of these peaks almost remained the same. Thus, it was reasonable to assume that three PL peaks came from the ground state transitions of InAs QDs with three different size distributions. It was suggested to prove this by cross-sectional transmission electron microscopy (XTEM). However, the strain field of an embedded QD makes the boundary between InAs QD and its surrounding materials very vague, so the XTEM cannot show the

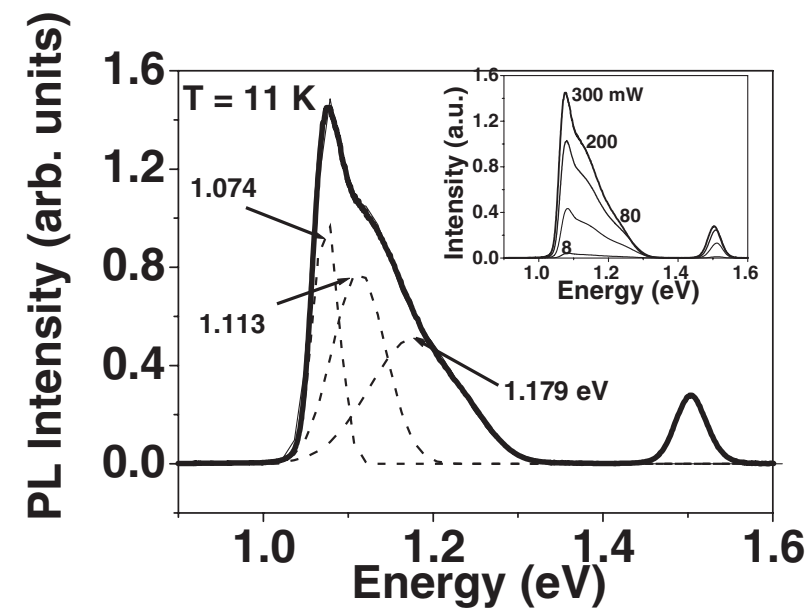

(a)

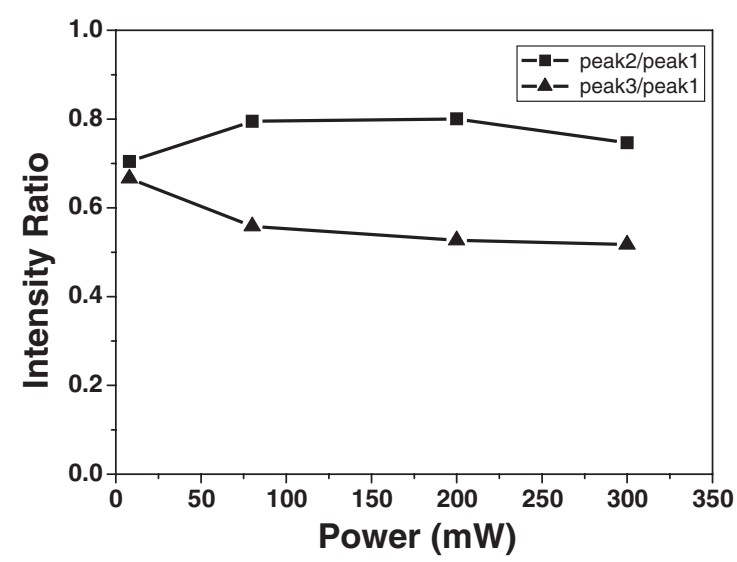

(b)

Fig. 3. (a) PL spectra of InAs/AlGaAs/GaAs QDIP. The spectra can be fitted by superposition of three Gaussian peaks at 1.074 (peak 1), 1.113 (peak 2), and $1.179 \mathrm{eV}$ (peak 3). The inset shows the PL spectra as a function of laser power from 8 to $300 \mathrm{~mW}$. (b) Normalized intensity ratio of peak 2 /peak 1 and peak $3 /$ peak 1 as function of laser power.

difference. Usually, other groups took $1 \mathrm{~nm}$ as the finest statistical measuring error. ${ }^{18,19)}$ Such an error is far beyond the difference in size distribution that leads to 39 and $66 \mathrm{meV}$ separations between the neighboring peaks. That is, the difference in average size responsible for peaks 1 and 2 


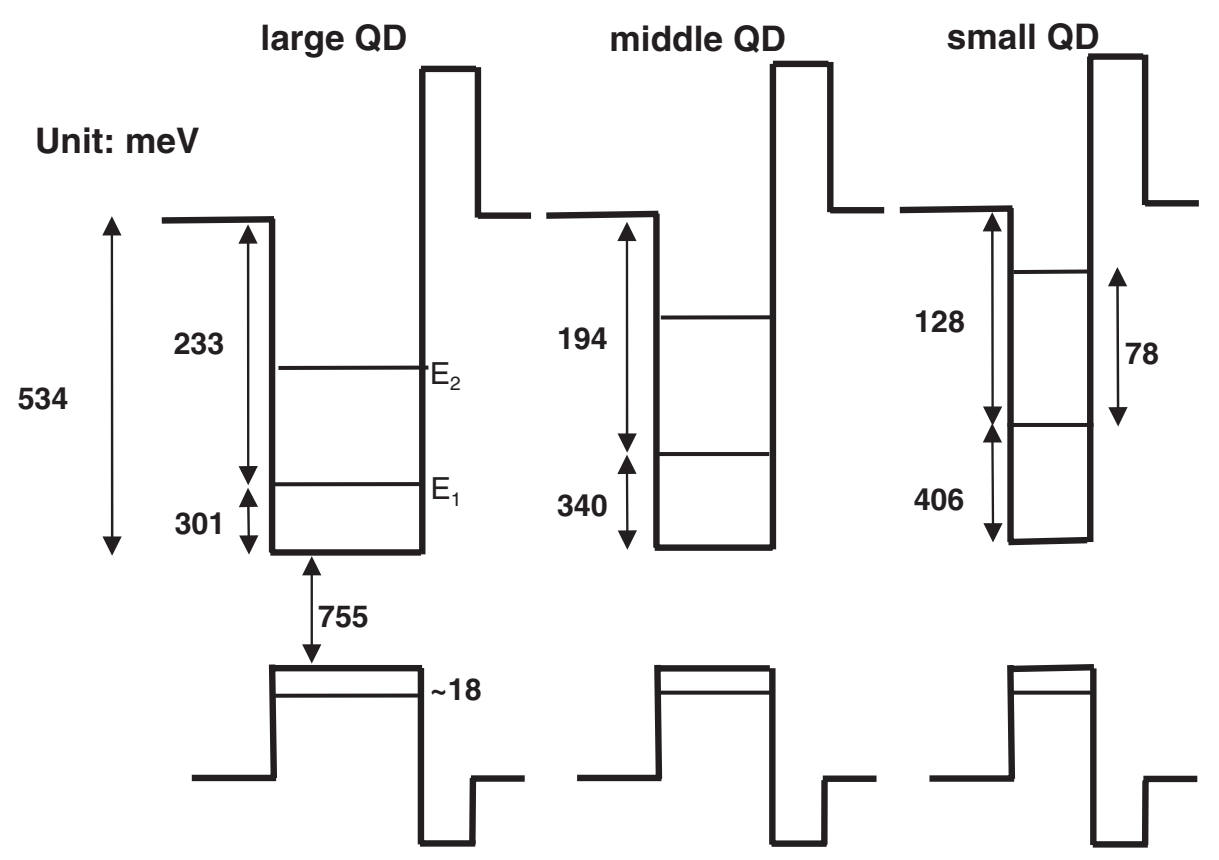

Fig. 4. Schematic band diagrams of large, medium and small InAs/AlGaAs/GaAs QDs fitted with PL and spectral response data.

is less than $1 \mathrm{~nm}$. Another noteworthy observation is that energy differences between the three peaks, i.e., 0.2296, 0.1938 and $0.1305 \mathrm{eV}$, in the spectral response of QDIP are the same as those of $1.074,1.113$ and $1.179 \mathrm{eV}$ in the PL spectra. According to our calculations, such difference comes mainly from different energy discontinuities between the electron ground state and GaAs conduction band, since the variations in hole energy states are very small. This implies that the three peaks of QDIP spectral response may result from the same transition mechanism, i.e., from the ground state to the GaAs conduction band.

In the case of conventional InAs/GaAs QDs, only a single broad PL spectrum was observed, indicating that the distributions of InAs QDs were broadened and merged with one another. Joyce et al. ${ }^{20)}$ observed the (In,Ga)As alloying of InAs QDs with the lower GaAs material on (001)GaAs. This was explained as the InAs/GaAs intermixing due to In segregation. This phenomenon causes the variation in In composition in QDs and increases PL linewidth. Arzberger et al. ${ }^{21)}$ proposed that the InAs/GaAs intermixing caused by In segregation at $T_{\mathrm{S}}=530^{\circ} \mathrm{C}$ can be considerably suppressed by a thin AlAs capping layer which in turn decreases PL linewidth. Other groups also observed this similar phenomenon when capping InAlAs at $T_{\mathrm{S}}=520^{\circ} \mathrm{C}$ and this phenomenon was explained by such a suppressing process. ${ }^{19,22)}$ However, here we propose another explanation. The melting point of AlGaAs is higher than that of GaAs. Therefore, AlGaAs can generate a higher latent heat than that of GaAs when from the gas state it condenses on the sample. The released latent heat loosens the bondings of the surface atoms to the InAs QDs and results in a stronger In/ $\mathrm{Al}$ intermixing than in the case of $\mathrm{In} / \mathrm{Ga}$. The degree of In/ $\mathrm{Al}$ intermixing on the different part of a QD is stress-driven. The stronger $\mathrm{In} / \mathrm{Al}$ intermixing tends to decrease to a greater extent the compressive strain of InAs QDs, which leads to a more uniform strain in InAs QDs. The behavior results in a small linewidth. Furthermore, we here propose another mechanism as a complement. After capping with a $2 \mathrm{~nm}$ $\mathrm{Al}_{0.3} \mathrm{Ga}_{0.7} \mathrm{As}$ layer, the high potential barrier of $\mathrm{AlGaAs}$ surrounding larger QDs exerts a lesser effect on the energy levels inside the dots than that surrounding smaller QDs, as shown schematically in Fig. 4. This means that the energy difference between the ground states $\mathrm{E}_{1}$ of large and small QDs increases after capping with the $2 \mathrm{~nm} \mathrm{Al}_{0.3} \mathrm{Ga}_{0.7} \mathrm{As}$ layer. Therefore, the emission peaks separate further apart and become distinguishable.

Since the higher bandgap AlGaAs cap layer covers the lower part of a QD, conduction electrons can only be transported through the top of the InAs QD that contacts the $\mathrm{GaAs}$ region. The photoresponse will be initiated when one electron makes a transition from the bound state to the conduction band of GaAs, not AlGaAs. The first excited state $E_{2}$ of the smallest QDs is pushed up to the brink of the GaAs conduction band edge; the electrons in this state have a higher probability of tunneling through the neighboring barrier when an electric field is applied. Although Movilla et $a l .{ }^{23)}$ theoretically showed that the effects of AlAs layers on the electronic states of QDs are quite weak, their model did not consider the change in shape or In composition of QD due to the In/Al intermixing. Therefore, the insertion of AlGaAs layer can still sufficiently change the electronic states in QDs.

AFM and scanning electron microscopy, which are not shown here, indicate that the height of InAs QD, i.e., $\sim 6 \mathrm{~nm}$, is much smaller than the lateral dimension, i.e., $30-50 \mathrm{~nm}$, which has only a weak quantum confinement. The main quantum confinement could be focused in the vertical direction. Therefore, the approximate simulation using a quantum well model is acceptable. The precise energy level of InAs QDs should be derived through a well-defined 3D model. The peak energies of PL and the spectral response peaks of QDIP can thus be fitted by the theoretical calculation of energy levels inside the QDs using the quantum well model, as shown in Fig. 4. In the calculation, 
Table I. Parameters used in theoretical calculation of energy levels in InAs/GaAs QDs. $m_{\mathrm{e}}^{*}$ and $m_{\mathrm{h}}^{*}$ are the effective masses of electrons and holes in the unit of free electron mass $m_{0} . E_{\mathrm{g}}$ is the band gap of GaAs and InAs under different strain conditions.

\begin{tabular}{lccc}
\hline & $E_{\mathrm{g}}(\mathrm{eV})$ & $m_{\mathrm{e}}^{*}\left(m_{0}\right)$ & $m_{\mathrm{h}}^{*}\left(m_{0}\right)$ \\
\hline GaAs & 1.518 & 0.067 & 0.51 \\
\hline Unstrained InAs & 0.418 & & \\
\cline { 1 - 2 } Partially strained InAs & $0.418-0.785$ & 0.023 & 0.41 \\
\cline { 1 - 2 } Fully strained InAs & 0.785 & & \\
\hline
\end{tabular}

the conduction band offset is set to be $70 \%$ of the band gap difference between InAs and GaAs and the strained InAs band gap is close to $0.755 \mathrm{eV}$. All the parameters used in the calculations are summarized in Table I. The ground state energies of holes are calculated to be about $18 \mathrm{meV}$ in three different sizes of QDs with very small variations. The $5.4 \mu \mathrm{m}$ $(230 \mathrm{meV})$ and $9.5 \mu \mathrm{m}(131 \mathrm{meV})$ peaks are due to the transitions of large and small QDs from the ground state to the GaAs conduction band, respectively. The response peak of medium QDs is about $6.4 \mu \mathrm{m}(194 \mathrm{meV})$ but embedded in the $\mathrm{H}_{2} \mathrm{O}$ absorption region.

The $15.8 \mu \mathrm{m}(78 \mathrm{meV})$ peak is suggested to originate from the bound-to-bound transition of small QDs from the ground state to the first excited state and then their tunneling out. In Fig. 2, a much smaller side peak at $18 \mu \mathrm{m}$ and a merged peak at $16.8 \mu \mathrm{m}$ appear near the $15.8-\mu \mathrm{m}$ main peak. These two peaks locate at the longer wavelength. They are attributed to the transitions of large and medium QDs from the ground state to the first excited state. Because the potential barriers between the first excited state and the GaAs conduction band are higher for larger QDs, the tunneling probabilities for medium and large QDs are lower than that for small QDs. The linewidth ratio $(\Delta \lambda / \lambda)$ of the large $15.8 \mu \mathrm{m}$ peak that is much smaller than those of the other two large peaks also indicates that it is responsible for the bound-to-bound transition while those of the other two large peaks are for bound-to-continuous transition. In ref. 15, the linewidth ratios of the two shorter wavelength peaks are 42 and $28 \%$, and that of the longest wavelength peak is also large, while those of ours are much smaller $(29,13$ and $8 \%)$. Considering the bound-to-bound transition, the AlGaAs-capped QDs have a larger barrier than the InGaAs-layer quantum-dotsin-a-well structure for electrons to tunnel through. The tunneling time of bound-to-bound transition electrons in our InAs/AlGaAs/GaAs QDIP, therefore, is longer than that in InAs/GaAs or InGaAs-sandwiched quantum-dots-ina-well QDIPs. According to the uncertainty principle $(\Delta E) *(\Delta t) \geq \hbar / 2$, energy dispersion in the InAs/AlGaAs/GaAs QDIP will be smaller, thus the smaller linewidth.

Another noteworthy feature of the InAs/AlGaAs/GaAs QDIP is its high responsivity. Compared with the conventional InAs/GaAs QDIP, at least one order of magnitude increase in responsivity was observed. Responsivity strongly depends on QD density, material quality of QDs and device structure. The comparisons of the two different QDIP structures were performed under the same growth conditions, which are expected to have the same material quality of QDs, except for the influence of different capping layers. In other laboratories, different growth conditions and device structures cause different characteristics of QDIP. For example, the authors of ref. 24 reported a responsivity of $4.9 \mathrm{~A} / \mathrm{W}$ at $10 \mu \mathrm{m}$, which corresponds to a quantum efficiency of $61 \%$. Such a high responsivity of the conventional $\mathrm{In}_{0.3} \mathrm{Ga}_{0.7}$ As QDIP may also originate from its high material quality, a higher dot density of $\sim 6.5 \times 10^{10} \mathrm{~cm}^{-2}$, a higher dopant concentration of two $\mathrm{Si}$ atoms per dot, more stacks of QD active layers, or even a thicker $50 \mathrm{~nm}$ GaAs spacer. In the theoretical studies by Ryzhii et al. ${ }^{10)}$ the rate of photoexcitation is given by $G^{\mathrm{ph}}=\sigma I \Sigma_{\mathrm{QD}} K N$, where $\sigma$ is the photoescape cross section, $I$ is the photon flux, $\Sigma_{\mathrm{QD}}$ is the sheet density of QDs in each QD array, $K$ is the number of QD arrays in the QDIP, and $N$ is the average number of electrons in QDs. To realize a higher responsivity, increasing the sheet density of QDs, number of QD arrays, or dopant concentration is then useful a method. Another key factor is the photoescape cross section $\sigma$. The insertion of the 2-nm-thick AlGaAs layer in the InAs/AlGaAs/GaAs QDIP affects the cross section. It changes the escape-and-recapture process of photoelectrons as a function of $\mathrm{Al}$ mole fraction and thickness. A high $\mathrm{Al}$ mole fraction forms a high barrier that will decrease tunneling probability, but prevents the recapture of photoelectrons by the wetting layer. A thicker AlGaAs layer completely covering the QDs also decreases tunneling probability and cannot increase the energy difference between small and large QDs as mentioned above. Thus, it is a trade-off in choosing between $\mathrm{Al}$ mole fraction and AlGaAs layer thickness.

In the InAs/GaAs QDIP, the photocarrier pumped out from QDs to GaAs conduction band can be recaptured by the wetting layer of the next stack of QDs, while in the InAs/ $\mathrm{AlGaAs} / \mathrm{GaAs}$ QDIP, the $2 \mathrm{~nm} \quad \mathrm{Al}_{0.3} \mathrm{Ga}_{0.7} \mathrm{As}$ potential barrier behind the wetting layer can reflect the carrier and decrease capture rate. The lifetime of the photocarrier is therefore increased and so is responsivity.

\section{Conclusions}

In conclusion, by introducing the $2 \mathrm{~nm} \mathrm{Al}_{0.3} \mathrm{Ga}_{0.7} \mathrm{As}$ cap layer on 3-ML InAs QDs, the responsivities of QDIP exhibited three large peaks, i.e., 5.4, 9.5, and $15.8 \mu \mathrm{m}$. The responsivities were quite high under the $-1.2 \mathrm{~V}$ bias, i.e., $0.79,0.47$, and $0.63 \mathrm{~A} / \mathrm{W}$. The two shorter-wavelength peaks are attributed to the transitions of large and small QDs from the ground state to the GaAs conduction band. The $15.8 \mu \mathrm{m}$ peak is attributed to the transition of small InAs QDs from the ground state to the first excited state followed by the tunneling out of QDs. The high responsivity is due to the increase of photocarrier lifetime by adding the AlGaAs potential barrier.

\section{Acknowledgement}

This work is supported by the National Science Council of Republic of China under contact No. NSC 91-2120-M002-002.

1) S. Z. Chang, T. C. Chang, J. L. Shen, S. C. Lee and Y. F. Chen: J. Appl. Phys. 74 (1993) 6912.

2) S. Z. Chang, T. C. Chang and S. C. Lee: Appl. Surf. Sci. 92 (1996) 70.

3) K. H. Schmidt, G. Medeiros-Ribeiro, U. Kunze, G. Abstreiter, M. Hagn and P. M. Petroff: J. Appl. Phys. 84 (1998) 4268.

4) L. Chu, M. Arzberger, G. Bohm and G. Abstreiter: J. Appl. Phys. 85 
(1999) 2355.

5) M. G. Alessi, M. Capizzi, A. S. Bhatti, A. Frova, F. Martelli, P. Frigeri, A. Bosacchi and S. Franchi: Phys. Rev. B 59 (1999) 7620

6) K. H. Schmidt, G. Medeiros-Ribeiro, J. Garcia and P. M. Petroff: Appl. Phys. Lett. 70 (1997) 1727.

7) S. F. Tang, S. Y. Lin and S. C. Lee: Appl. Phys. Lett. 78 (2001) 2428.

8) S. Y. Lin, Y. R. Tsai and S. C. Lee: Appl. Phys. Lett. 78 (2001) 2784.

9) J. D. Phillips: J. Appl. Phys. 91 (2002) 4590.

10) V. Ryzhii, I. Khmyrova, V. Mitin, M. Stroscio and M. Willander: Appl. Phys. Lett. 78 (2001) 3523.

11) Q. Xie, A. Kalburge, P. Chen and A. Madhukar: IEEE Photonics Technol. Lett. 8 (1996) 965.

12) F. Y. Chang, C. C. Wu and H. H. Lin: Appl. Phys. Lett. 82 (2003) 4477.

13) H. Petterson, L. Baath, N. Carlsson, W. Seifert and L. Samuelson: Appl. Phys. Lett. 79 (2001) 78.

14) S. Y. Lin, Y. J. Tsai and S. C. Lee: Jpn. J. Appl. Phys. 40 (2001) L1290.

15) S. Krishna, S. Raghavan, G. von Winckel, A. Stintz, G. Ariyawansa,
S. G. Matsik and A. G. U. Perera: Appl. Phys. Lett. 83 (2003) 2745.

16) H. C. Liu, M. Gao, J. McCaffrey, Z. R. Wasilewski and S. Fafard: Appl. Phys. Lett. 78 (2001) 79.

17) S. Kaiser, T. Mensing, L. Worschech, F. Klopf, J. P. Reithmaier and A. Forchel: Appl. Phys. Lett. 81 (2002) 4898.

18) Yu. I. Mazur, Z. M. Wang, G. J. Salamo, Min Xiao, G. G. Tarasov, Z. Ya. Zhuchenko, W. T. Masselink and H. Kissel: Appl. Phys. Lett. 83 (2003) 1866

19) R. Jia, D. S. Jiang, H. Y. Liu, Y. Q. Wei, B. Xu and Z. G. Wang: J. Cryst. Growth 234 (2002) 354.

20) P. B. Joyce, T. J. Krzyzewski, G. R. Bell, B. A. Joyce and T. S. Jones: Phys. Rev. B 58 (1998) 15981.

21) M. Arzberger, U. Kasberger, G. Bohm and G. Abstreiter: Appl. Phys. Lett. 75 (1999) 3968.

22) Z. Y. Zhang, B. Xu, P. Jin, X. Q. Meng, Ch. M. Li, X. L. Ye and Z. G. Wang: J. Appl. Phys. 92 (2002) 511.

23) J. L. Movilla, J. I. Climente and J. Planelles: J. Appl. Phys. 94 (2003) 4515.

24) D. Pal, L. Chen and E. Towe: Appl. Phys. Lett. 83 (2003) 4634. 